\title{
Control of Electric Drives in the Best Efficiency Region of Pumping System
}

\author{
Ilja Bakman (Doctoral Student, Tallinn University of Technology)
}

\begin{abstract}
The paper concentrates on improving the efficiency of the pumping system in which pumps are run by variable speed drives. The method is applicable to system consisting of several centrifugal pumps. Problems arising from running the pumps outside the optimal efficiency region are discussed. Common principles of operation and control of typical boosting pumping station are represented. Methods of regulation of productivity of the system are described. The proposed method is based on prediction of future efficiency of pumps before making adjustments of pumping system productivity. The proposed method is basing on tracking the working point of pump on efficiency graph. Monitoring the location of working point vs. efficiency graph enables to evaluate its current and future distances from the best efficiency point of the pump. Knowing the current number of running pumps it is possible to predict the location of working point after adjustment of productivity of the system. Ability to predict the increase or decrease of efficiency enables to enhance the adjustment of productivity of the system. Methods of graphical analysis of working state of the pumping system are representing topic for future research.
\end{abstract}

Keywords - Fluid flow control; Pumps; State estimation; Variable speed drives.

\section{INTRODUCTION}

According to the results of research, pumps consume no less than $10 \%$ of electrical power produced worldwide [1]. Due to the developments of power electronics, the efficiency of modern pumping systems has increased. Today, however, pumping that consumes a substantial share of produced power is still a subject to technological upgrade.

Development of powerful programmable controllers enables to enhance the efforts of increasing the efficiency of pumping stations by applying the flexible control algorithms, data acquisition and signal processing.

Various methods and solutions are applied in order to improve the efficiency of pumps driven by electric motors. Efficiency growth of the pumping systems helps to save the expenses in both the power consumption and the maintenance. Additionally, the length of lifecycle depends on the grade of efficiency indexes during the pump usage.

Modern equipment built on the basis of the variable speed drives (VSD) can significantly increase the efficiency of pumping stations. The variable speed pumping has shown its advantages over throttling pumping, which was widely used before the development of power electronic devices and digital signal processors because of its simple and low-cost implementation. The main advantage of the VSD over the throttling system is the elimination of the power dissipation in the piping system resulting from chocking the discharge.

However, difficulties arise when a pump driven by the VSD is forced to run with a non-rated speed outside the best efficiency region (BER). In such cases the efficiency of the whole pumping unit decreases. The decrease is particularly sharp in the speed range below the rated speed [2], [3]. Such problem is likely to occur in multi-pump systems as a result of over- (or under-) sizing on the design stage or abnormal demand conditions, which can be caused by some defects of the piping system [4].

Typically the control system adjusts the rotational speed to provide a constant supply when the demand is changing, in the traditional pumping stations where pumps are running with VSD. The pumps are being turned on and off in order to keep the supply on the desired level. The speed variations in such kind of systems can bring the pump to functioning outside the BER [5].

This paper focuses on the pumping systems consisting of centrifugal pumps fed by separate VSDs. A new method of efficiency improvement for the centrifugal pumps running in the multi-pump applications is proposed. This approach is based on the estimation of the future performance of pumps before turning the additional pumps on or off. Comparing the performances before turning on and off should help to avoid pump performance at a low efficiency level due to running of excess pumps. Additionally, a method of prediction of the future efficiency resulting from the number of working pumps changing is discussed.

\section{PUMPING SYSTEM}

The multi-pump system provides the following benefits against system containing of one large pump [6]:

- Redundancy. The supply can be provided even if one or several pumps are out of order. Failure of one or several pumps can affect the performance of the system but will not cause the breakdown of the whole pumping station.

- Reducing the costs connected to keeping the spare parts and units.

- Flexibility. The desired pressure can be provided by varying the number of working pumps which increases the probability of functioning closer to the BER as compared to the use of one large pump. Functioning in the BER ensures saving both the energy costs and the maintenance costs.

Most of the pumping applications (excluding the levelcontrolled systems) are designed to maintain the constant pressure in the pumping station outlet [7]. The pressure constancy is achieved by adjusting the produced flow, which can be implemented by varying the rotational speed of the pump. 
Pressure at the outlet of the pumping station is adjusted in order to follow the set-point. In this way, compensation of the pressure decrease is implemented through the increase of flow.

The control system is measuring the feedback (typically a water pressure at specific section of pipeline). In order to bring the output pressure to conformity with the set-point, the control system can:

- adjust the rotational speed of a pump

- affect the flow by changing the number of running pumps through turning on and off the units

Adjustment of the rotational speed of the pump is based on the PID regulation principle. Here the set-point for the PID controller is a desired pressure set by an operator. The feedback is the water pressure acquired from a pressure sensor at the output of the pumping system. The PID controller provides a speed reference to the VSD in order to compensate the difference between set-point and actual pressure at the output of the system [8]. The PID controller is implemented as a software module inside the controller which runs the pumping system [9].

Extra flexibility of productivity is achieved by increasing or decreasing the number of working pumps in the system. The decision on changing the number of working pumps is typically made when the actual speed reference generated by the PID controller overcomes the predefined limit.

If the speed reference for pumps grows significantly it means that given number of working pumps cannot provide enough flow. So as the limit is overcome, the next pump is turned on. If the speed reference for pumps decreases significantly it means that the given number of pumps provides too much flow. Hence number of working pumps can be decreased. When the limit is overcome, the next pump is turned off. This principle of management of number of running pumps is shown in Fig. 1.

Managing the multi-pump system in which every pump is run by a separate VSD requires the use of industrial communication equipment. The messages from the network master can contain the start and stop commands and the speed references for pumps. Here all pumps run with same speed. This is a common practice in parallel pumping systems for avoiding the flow recirculation. Each unit of the multi-pump system consists of the VSD, asynchronous motor, and the pump. The PLC as the network master runs the PID controller and generates the turn off and on commands. The structure of the described multi-pump system is shown in Fig. 2.

When the pumps are connected in parallel, they act against the common pressure. In this case the total flow rate consists of the sum of flow rates of all pumps. When the pumps are connected in series, the total pressure at the system output is a sum of pressures provided by every single pump.

Further in this paper only the parallel connection will be discussed.

Multi-pump operation is sensitive to the individual characteristics of each pump. The total pressure may not be a simple sum of individual pressures, especially in the cases when characteristics of the pumps are not similar.

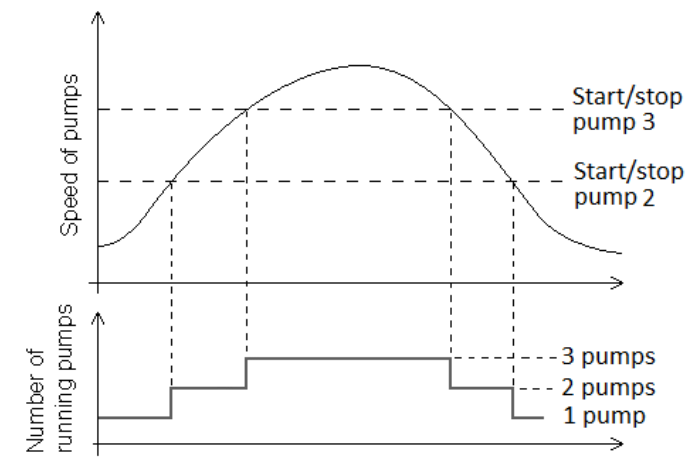

Fig. 1. Principle of adjusting the number of working pumps dependently on speed.

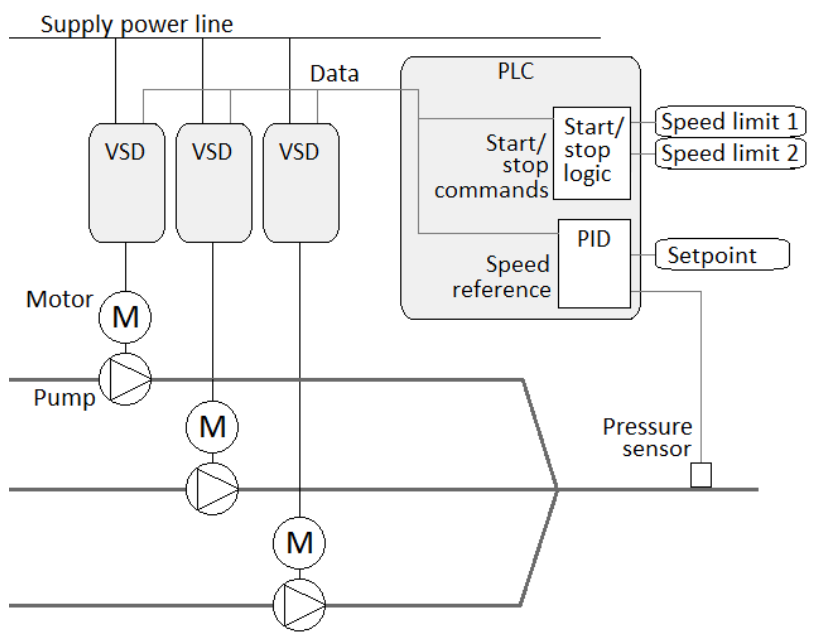

Fig. 2. Structure of a typical multi-pump system.

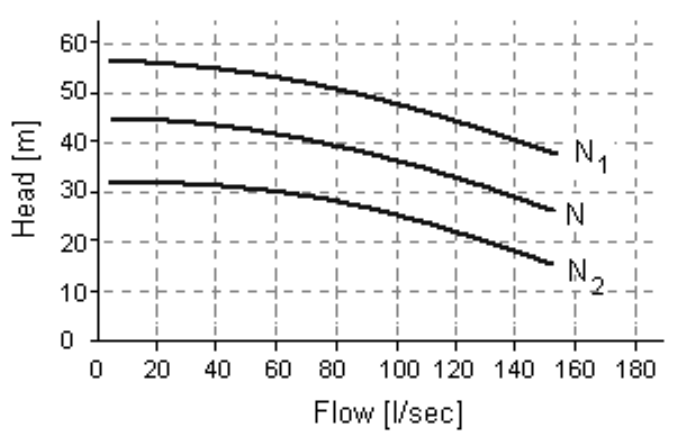

Fig. 3. Pump curves of Ebara $120 \mathrm{CDX}$ centrifugal pump ( $N$ - rated speed, $N_{l}>N>N_{2}$ ).

To achieve the BER, all the pumps could be running with the VSDs following the speed reference of the master [10]. However, in some applications all pumps except one are running at a constant speed whereas one of the pumps is running at a variable speed, thus adjusting the flow.

\section{PUMP CURVES}

The characteristics of the pumps are given on the diagrams known as the pump curves. These diagrams supplied by the manufacturers are individual for every pump model. The main pump characteristic is the head-flow curve, which shows the relation between the flow (amount of a fluid moved per unit time) and the head (measure of pressure provided by the pump). For all centrifugal pumps it is typical that the flow rate decreases as the head increases (Fig. 3). 
Several characteristic curves represent the pump's performance at various speed rates.

Changing the speed of a pump affects the rates of flow and the head. The characteristic curves change according to affinity laws [11] as follows:

$$
\frac{Q_{1}}{Q_{2}}=\frac{N_{1}}{N_{2}}
$$

and

$$
\frac{H_{1}}{H_{2}}=\left(\frac{N_{1}}{N_{2}}\right)^{2},
$$

where

$Q$ - flow rate, $\mathrm{m}^{3} / \mathrm{s}$,

$N$ - speed of pump, rpm,

$H$ - actual head of pump, m.

index 1 represents the initial state and index 2 - the next state.

The system resistance curve (or system curve) represents the resistance of the piping system and depends on consumers. The generic shape and slope of the system curve depends on a specific application and is not provided with the datasheets of the pump. It varies when the structure of the consumer or the pipeline changes. Variation in the household daily consumption is one of the factors affecting the shape of the system curve. The system curve tends left when the resistance is high; it tends right when the resistance falls. The point of crossing of the head-flow curve with the system curve indicates the working point of the pump.

When the discharge valve of the pump is closed, the provided head is at its maximum and the flow is at zero point.

The pump size and type are selected on the basis of the characteristics of the system curve. Knowledge of the working point location on the system curve helps to determine if the pump is able to provide enough head and flow by re-uniting the pump characteristic curve with the system curve.

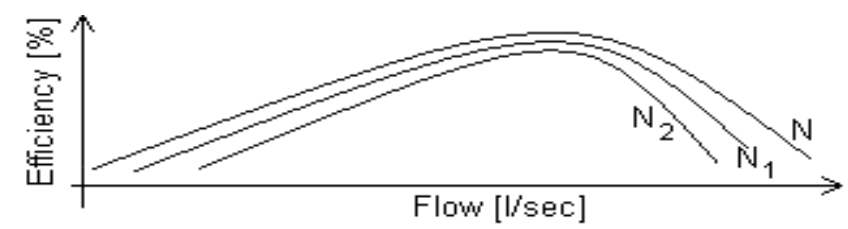

Fig. 4. An efficiency curve at the rated speed $\left(N-\right.$ at rated speed, $N_{l}, N_{2}-$ at non rated speeds).

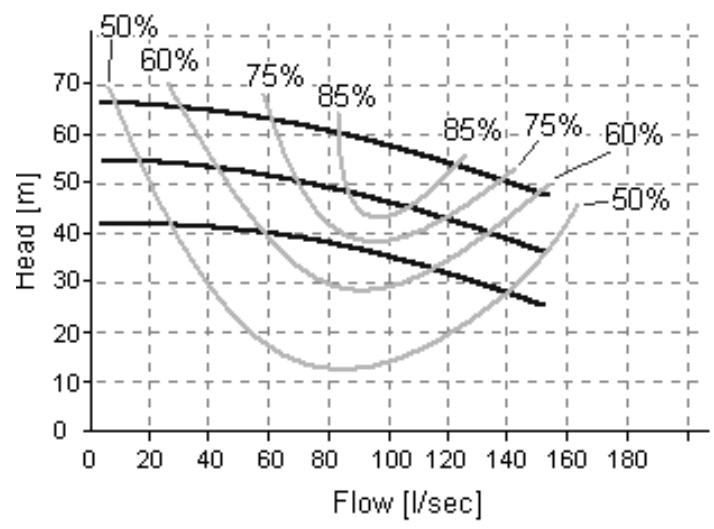

Fig. 5. Efficiency map: head-flow and efficiency curves.
Another important characteristic of the pump is its efficiency curve. One of them for Ebara $120 \mathrm{CDX}$ pump is shown in Fig. 4.

The point at which the efficiency curve is rising to its peak is known as the best efficiency point (BEP).

Taking into consideration the fact that efficiency decreases at non-rated operation, the efficiency curves can be drawn for every speed rate shown in Fig. 3.

The projection of the efficiency curve set onto the headflow graph results in an efficiency map [12].

By combining Figs. 3, 4 and 5 and observing the relationship between several speed curves, the efficiency of the particular pump, and the efficiency map, it is possible to obtain the resulting curve representing the efficiency at the rated speed forming the BER.

\section{RECOMMENDED OPERATION REGIONS FOR VSD DRIVEN PUMPS}

Pump operation exactly in its BEP is almost impossible due to variations of the system curve. Thus, operation as close to the BEP as possible is a target. Therefore, the BER is the most preferable operating space.

It is highly recommended to run a centrifugal pump in the BER for optimizing the pump efficiency and its energy consumption. Also, such harmful phenomena as cavitation and hydraulic excitation forces are minimized in the BER [5], which provides higher reliability and longer lifecycle.

Operation far from the BER is accompanied with such abnormal phenomena as Flow recirculation (leads to reduced output flow), reduced bearing and sealing life, cavitation and temperature increase of the pumped fluid [6].

Knowing that operating at the BEP is almost impossible and the probability of overcoming the limits of the BER always exists, the Preferable Operating Region (POR) and Allowable Operating Region (AOR) were introduced [7].

According to [13], $70 \%$ to $120 \%$ of the flow rate at the BEP is preferable for a typical radial flow centrifugal pump. AOR includes a wider range of flows. Operation in this region should not lead to immediate failure of a pump; however its lifecycle will be shortened. The hazards here are of the higher rates of vibration and noise as compared to POR. Working in AOR (which is $70 \%$ to $50 \%$ of the flow rate at the BEP) leads to inefficient pumping but does not harm the pump in a direct manner. Thus, operating in the AOR region is possible for a short time. In total, it can even reach hundreds of hours per lifecycle [14] and should not be treated as forbidden.

\section{ENERGY CONSUMPTION OF A PUMP}

Amount of the consumed electrical power depends on the electrical parameters and conditions of functioning of the pump and its drive. Here, the term drive includes a motor and a feeding device (VSD). In this section, the multi-pump system consisting of the pumping sets is described each of which consists of a pump and its drive. The pumps in the multi-pump system are supposed to have identical characteristics of flow $(Q)$ and head $(H)$. 
An effective operation of the multi-pump system can be provided by the control system. The components of the pumping system (and mainly the control system) ought to keep the consumption of electricity on a rational level. This is intended to be reached by minimizing the momentary value of electric power consumed by the pumping system.

Electric power being consumed by the pumping system can be defined as [15]:

$$
P_{e l}=\sum_{i=1}^{m} \frac{\rho \cdot g \cdot Q_{i} \cdot H_{i}}{\eta_{z i}},
$$

where:

$$
\eta_{z i}=\eta \cdot \eta_{s} \cdot \eta_{f}
$$

$\eta$ - pump efficiency

$\eta_{f}-$ efficiency of VSD

$\eta_{s}$ - efficiency of induction motor

$\eta_{z}$ - efficiency of pumping set

$\rho$ - density of pumped fluid, $\mathrm{kg} / \mathrm{m}^{3}$,

$m$ - number of working pumps

The presence of the flow-head product in (3) confirms the dependence of consumed electrical power on the working point.

\section{VARYING THE NUMBER OF WORKING PUMPS AND ITS IMPACT ON EFFICIENCY}

As it was stated earlier, one of the preferable methods of the control of pump productivity is by varying its speed. The productivity of a full pumping system (consisting of several pumps) can be adjusted by varying the number of running pumps.

Typically at the stage of commissioning the principles of regulation of the running pumps number are defined. Most commonly the method of varying the number of running pumps is rated-speed oriented [16]. It means that an application is programmed in such a way that the number of pumps increases when the actual speed exceeds the predefined limit ("Start limit" in Fig. 1.). Similarly, the number of running pumps must decrease when the actual speed falls below the predefined limit ("Stop limit" in Fig. 1.).

The influence of turning on the additional pumps can be described by the dependency shown in Fig. 6 .

Thus, it is assumed that an increase of speed is caused by insufficient water supply (PID controller increases the speed reference in order to compensate the lack).

As an example, the pumping system consisting of two pumps can be considered.

Initially one pump can provide the desired supply. But if the demand grows, the PID controller should increase the speed reference. The next pump will be turned on as soon as the speed of other pumps overcomes the "Start limit".

As can be seen from Fig. 7, the characteristic curve shifts upwards. The resulting curve reflects the performance of the pump in the double-pump system. This shift of the performance curve cannot be described by the affinity law (1).

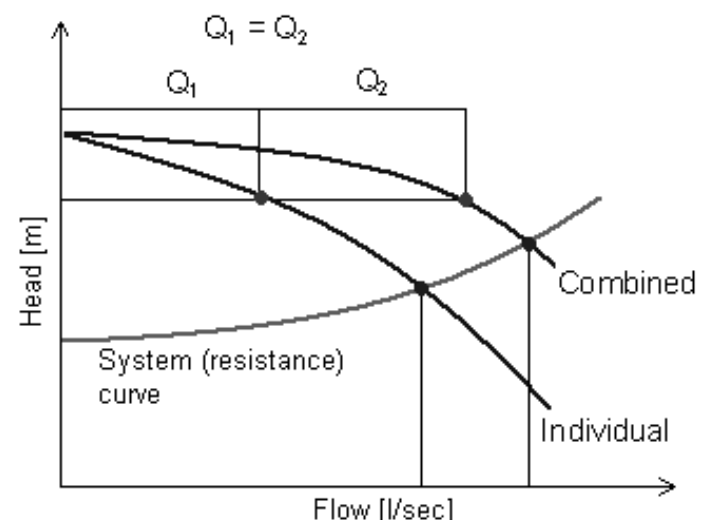

Fig. 6. Parallel operation of two pumps with similar rated parameters.

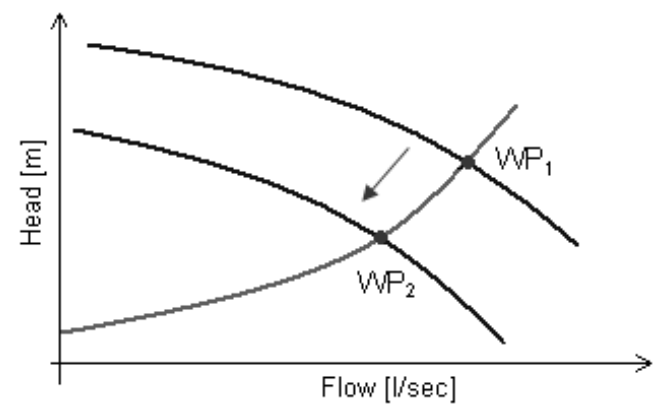

Fig. 7. Shifting of the operation point as a result of increasing the number of working pumps. The diagram reflects the state of one pump $\left(W P_{1}-\right.$ working point before start of second pump, $W P_{2}-$ working point after the start of second pump).

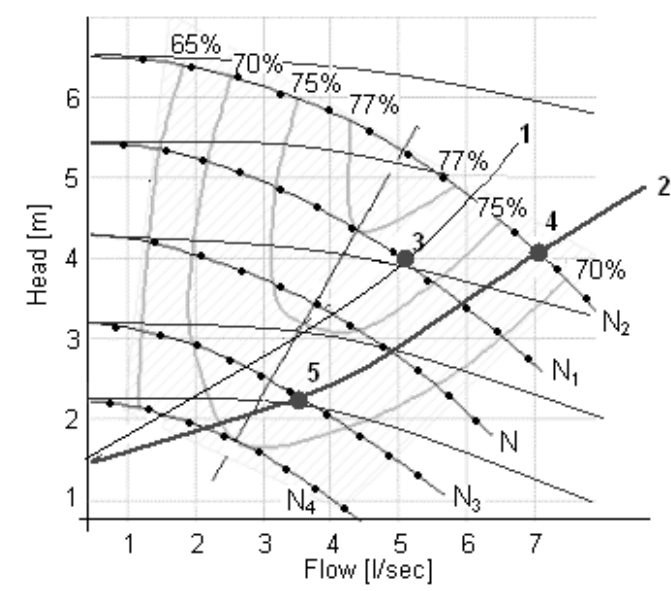

\footnotetext{
-0- Pump 1performance curve

Pump 1 and Pump 2 combined performance curve

1 - initial system curve

2 - new working curve

3 - initial working point

4 - new working point for 1 pump

5 - new working point for 2 pumps

$N$ - Performance curve at rated speed

$N_{1}, N_{2}$ - Performance curve at speed $>$ rated speed

$N_{3}, N_{4}$ - Performance curve at speed < rated speed
}

Fig. 8. Principle of comparing the actual and future distances from the BEP.

It is reasonable that the speed reference generated for each pump by the PID controller will be lower than the speed reference for one pump before the increment. One of the main tasks during the commissioning is to ensure that the speed reference after turning on the second pump will not slow down 
drastically (because of excess pressure) and will not result in turning the pump off (because of overcoming the "Stop speed" limit).

Naturally, such varying the number of running pumps affects the location of the working point relative to the BEP.

An increase or decrease of the number of running pumps is justified by the needs of keeping the speed in close-to-rated region. Also, it will certainly lead to shifting the operation point [17]. In the worst case such an increase can lead to shifting the working point out of AOR.

It is possible to state that the speed-oriented approach to the productivity control does not take into account the factors of the BER [18]. Also, the actions of the control system can harm the efficiency of the pump (though it keeps the supply at the desired level).

TABLE I

LOOKUP TABLE FED TO THE CONTROL SYSTEM

\begin{tabular}{|l|c|c|c|}
\hline \multirow{2}{*}{$\begin{array}{l}\text { Points } \\
\text { describing } \\
\text { the region }\end{array}$} & $77 \%$ & $75 \%$ & $70 \%$ \\
\cline { 2 - 4 } & {$[1.1]$} & {$[2.1]$} & {$[3.1]$} \\
\hline Point 1 & {$[1.2]$} & {$[2.2]$} & {$[3.2]$} \\
\hline Point 2 & {$[1.3]$} & {$[2.3]$} & {$[3.3]$} \\
\hline Point 3 & - & {$[2.4]$} & {$[3.4]$} \\
\hline Point 4 & - & {$[2.5]$} & {$[3.5]$} \\
\hline Point 5 & - & {$[2.6]$} & {$[3.6]$} \\
\hline Point 6 & & & \multicolumn{3}{|c|}{ Region of efficiency } \\
\hline
\end{tabular}

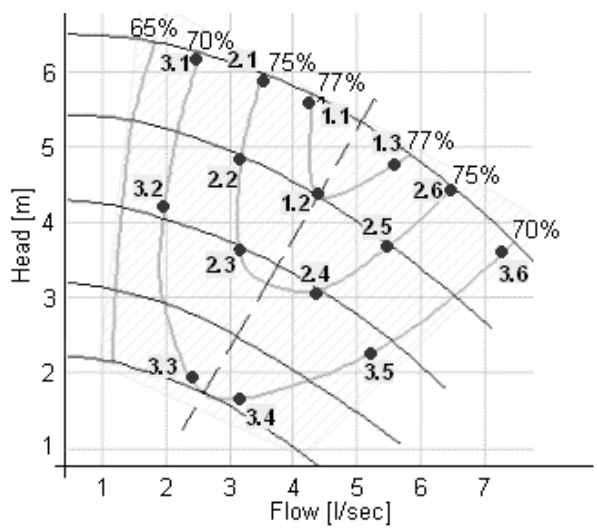

Fig. 9. The points describing the efficiency map.

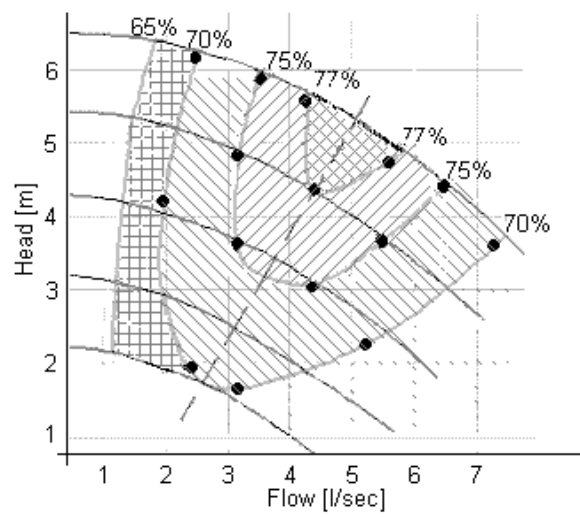

Fig. 10. Regions of pump efficiency as they should be seen by the detecting software. Various fillings reflect the regions of efficiency.
Fig. 8 describes the growth of the number of working pumps at the speed increase of an existing pump (or pumps) in order to keep the supply. One working pump is assumed as an initial condition. It can be seen that as soon as the system curve shifts right (the demand increases), there are two ways to keep the supply:

1. Turning on the auxiliary pump. The working point will shift to the location marked as " 5 ".

2. Increasing the speed of a single pump in order to increase the flow. In this case, the working point will shift to location " 4 ".

Fig. 8 shows that point " 4 " is closer to the BEP than point " 5 ". Hence increasing the speed of single pump is more benefit than turning on the second pump from the point of view of pump's efficiency. Here the trade-off takes place between the speed and the pump efficiency. The development of the trade-off strategies is a subject of future research.

Analysis of the future location of the working point and its impact on efficiency is difficult because of the amount and complexity of lines contained in the efficiency map. For an on-line analysis the map should be fed to the control system as a lookup table (Table I, Fig. 9 and Fig. 10).

The quality of the analysis will depend on the amount of points describing the lines of the efficiency map.

Detecting the location of actual working point and predicting the location of future working point with changed amount of working pumps can be implemented by the onboard DSP of the VSD's control unit [19]. The content of lookup table should be fed to the DSP as parameters.

TABLE II

LOOKUP TABLE OF THE CONTROL SYSTEM

\begin{tabular}{|c|c|c|c|}
\hline & $\begin{array}{c}\text { High } \\
\text { efficiency }\end{array}$ & $\begin{array}{c}\text { Middle } \\
\text { efficiency }\end{array}$ & $\begin{array}{c}\text { Low } \\
\text { efficiency }\end{array}$ \\
\hline Point 1 & {$[1.1]$} & {$[2.1]$} & {$[3.1]$} \\
\hline Point 2 & {$[1.2]$} & {$[2.2]$} & {$[3.2]$} \\
\hline Point 3 & {$[1.3]$} & {$[2.3]$} & {$[3.3]$} \\
\hline
\end{tabular}

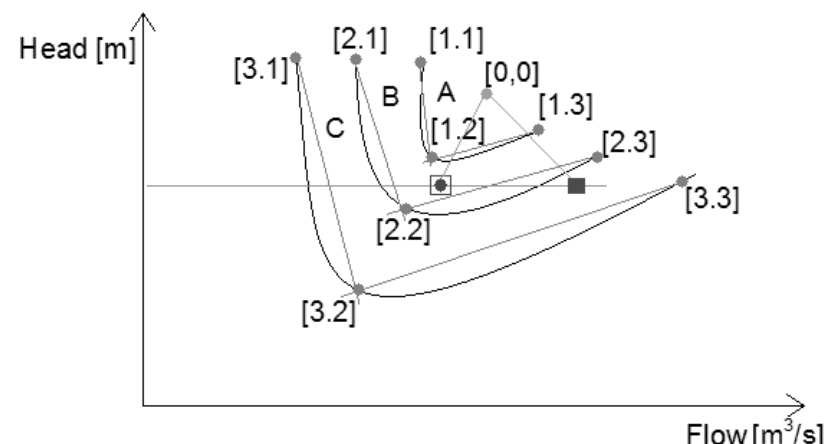

Current working point

- Future working point

- Distance from the center of most efficient range

Fig. 11. Marking of the lookup table. 
Location of working point is actually its affiliation to some efficiency region (e.g. $75 \%-77 \%$ ). The algorithms of detection will include analysis of the geometry of the efficiency map.

Acquiring the actual working point can be implemented using the algorithm of sensorless pressure control described in [10]. The algorithm enables to detect the location of actual working point utilizing the readings of pump input power which can be retrieved from the VSD's actual parameters.

For online analysis, the efficiency map similar to those shown in Fig. 8 is to be introduced to the control system as a lookup table (Table II and Fig. 11) [20].

The lookup table contains marker points of the efficiency curves which for simplicity of computations can be represented as a series of lines. This assumption is possible since the shapes of efficiency curves are alike and these curves do not intersect.

For example, define the efficiency curve 1 (range A) by 3 points: [1.1], [1.2] and [1.3]. The line [1.1]-[1.2] is determined as a function $\mathrm{f}_{1 \mathrm{~A}}(\mathrm{Q})$ represented by a line equation

$$
H=m \cdot Q+n,
$$

where

$m, n-$ are the line coefficients,

$Q-$ actual flow rate, $\mathrm{m}^{3} / \mathrm{s}$,

$H$ - actual head of pump, m.

The line [1.2] - [1.3] is determined as the similar function $f_{2 A}(Q)$. The line [2.2] - [2.3] will be determined as the function $f_{2 B}(Q)$, etc.

Finding of the working point location relatively these lines is a typical problem of analytical geometry on the plane. The distance from the center of the most efficient range ([0.0] in our case) is an important index. Combining these distances, the preferable efficiencies are calculated. The quality of the analysis and control depends on the amount of points described the lines of the efficiency map. The more is the number of marker points (lines), the higher is the approximation accuracy.

\section{CONCLUSIONS}

The method of the optimization of the control of the pump station productivity is proposed. The proposal offers a principle for the comparison of working point locations before and after the number of working pumps change. This comparison can be implemented on the basis of the future working point location estimation. Quite laborious parameterization of the control system is required to supply the data needed for comparison.

The algorithm for automatic comparison and decision making is a subject for further investigation and development.

\section{ACKNOWLEDGMENT}

This research work has been supported by the Estonian Ministry of Education and Research (Project SF0140016s11) and European Social Fund (project "Doctoral School of Energy and Geotechnology II").

\section{REFERENCES}

[1] IEA Report 2009, IEA Statistics, CO2 Emissions from fuel combustion.

[2] I. I. Ionel, Pumps and Pumping with Particular Reference to VariableDuty Pumps, Amsterdam: Elsevier, 1986, p. 715.

[3] V. Vodovozov, I. Bakman. (2013), "Slip Compensation in Sensorless Scalar Electric Drive of a Pump," $54^{\text {th }}$ International Scientific Conference of Riga Technical University. Riga: Riga Technical University, 2013, pp. 27.1-27.4.

[4] T. Ahonen. Monitoring of Centrifugal Pump Operation by a Frequency Converter, Doctoral Thesis, Lappeenranta University of Technology, 2011, p. 134.

[5] L. Szychta, "Analysis of efficiency characteristics of squirrelcage induction motor for pump applications," $14^{\text {th }}$ International Power Electronics and Motion Control Conference EPE-PEMC 2009, pp. 73 - 78.

[6] R. C. Dorf, Modern Control Systems, Prentice Hall, 2001, 831 p.

[7] I. J. Karassik and T. McGuire, Centrifugal Pumps, NY, USA: Chapman \& Hall, 1998.

[8] M. Pechenik, O. Kiselychnyk, S. Buryan and D. Petukhova, "Sensorless control of water supply pump based on neural network estimation," www.nbuv.gov.ua/portal/natural/emeo/2011_79/462-466.pdf.

[9] Z. Raud, V. Vodovozov and M. Egorov, "A toolbox to design and study electric drives," $14^{\text {th }}$ International Power Electronics and Motion Control Conference EPE-PEMC 2010, Ohrid, Macedonia, 2010, pp. T5-142-T5-148.

[10] V. Vodovozov, I. Bakman, "Sensorless pressure control of centrifugal pumps," $8^{\text {th }}$ International Conference-Workshop on Compatibility and Power Electronics CPE 2013, Ljubljana, Slovenia, 2013, pp. 304-309.

[11] V. Vodovozov, I. Bakman, "Control of liquid density to prevent abnormal pumping performance," $7^{\text {th }}$ WSEAS International Conference on Waste Management, Water Pollution, Indoor Climate WWAI 2013, Lemesos Cyprus, 2013, pp. 217-222.

[12] ABB Product Notes: Flow Calculation in ABB Industrial Drives, ABB, Helsinki, Finland, 2006.

[13] T. Ahonen, J. Tamminen, J. Ahola and J. Kestilä, "Frequency-converterbased hybrid estimation method for the centrifugal pump operational state,“ IEEE Transactions on Industrial Electronics, vol. 59, no. 12, 2012, pp. 4803-4809. http://dx.doi.org/10.1109/TIE.2011.2176692

[14] Z. Raud, V. Vodovozov, T. Lehtla and E. Pettai, "Simulation tools to study power electronics," $13^{\text {th }}$ European Conference on Power Electronics and Applications EPE 2009, Barcelona, Spain, 2009, paper 0013.

[15] F. Abrahamsen, F. Blaabjerg, J. K. Pedersen, P. Z. Grabowski and P. Thøgersen, "On the energy optimized control of standard and high efficiency induction motors in CT and HVAC applications," IEEE Transactions on Industrial Applications, v. 34, n. 4, 1998, pp. 822-831. http://dx.doi.org/10.1109/28.703985

[16] ABB, Technical Guide No. 1: Direct Torque Control, ABB, Helsinki, Finland, 2002

[17] IEC 60034-2-1: Rotating Electrical Machines - Part 2-1: Standard Methods for Determining Losses and Efficiency from Tests (Excluding Machines for Traction Vehicles), 2007.

[18] P. Girdhar, Centrifugal Pumps, Design, Operation and Maintenance, London: Elsevier, 2005, p. 244.

[19] ABB, ACQ810 Firmware manual, ABB, Helsinki, Finland, 2012.

[20] I. Bakman, L. Gevorkov, V. Vodovozov, "Predictive Control of a Variable-Speed Multi-Pump Motor Drive," 2014 IEEE 23 rd International Symposium on Industrial Electronics (ISIE 2014), Istanbul, Turkey, June 1st to 4th, 2014.

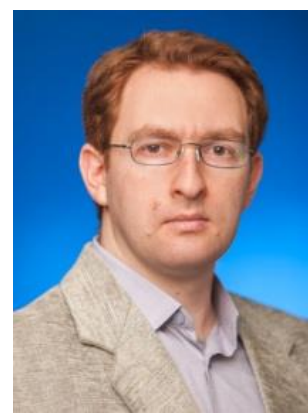

Ilja Bakman, 01.08.1981, Tallinn, Estonia. 2001-2005 Negev Academical College of Engineering, Ber-Sheva, Israel. Department of Power Electronics. B. Sc. degree. 2008-2012 Tallinn University of Technology, Faculty of Power Engineering, Department of Electrical Drives and Power Electronics. Master degree. 2012 - PhD studies at Tallinn University of Technology, Faculty of Power Engineering, Department of Electrical Engineering. 2008 Software developer, engineer in ABB AS, Tallinn, Estonia. Department of industrial projects.

Address: Tallinn University of Technology,

Ehitajate tee 5, Tallinn, 19086, Estonia

E-mail: ilja.bakman@gmail.com 